\title{
Directly targeting c-Myc contributes to the anti-multiple myeloma effect of anlotinib
}

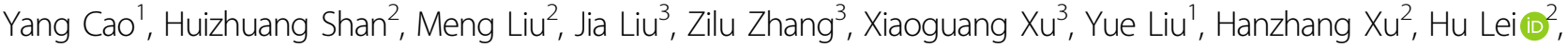 \\ Miao $\mathrm{Yu}^{2}$, Xingming Zhang ${ }^{2}$, Wanting $\mathrm{Liu}^{2}$, Zhilei Bu², Zhixiao Fang ${ }^{2}$, Yanjie $\mathrm{Ji}^{2}$, Hua Yan ${ }^{3}$, Weiying Gu (1) and \\ Yingli Wu (iD ${ }^{2}$
}

\begin{abstract}
Despite the significant advances in the treatment of multiple myeloma (MM), this disease is still considered incurable because of relapse and chemotherapy resistance, underscoring the need to seek novel therapies with different mechanisms. Anlotinib, a novel multi-targeted tyrosine kinase inhibitor (TKI), has exhibited encouraging antitumor activity in several preclinical and clinical trials, but its effect on MM has not been studied yet. In this study, we found that anlotinib exhibits encouraging cytotoxicity in MM cells, overcomes the protective effect of the bone marrow microenvironment and suppresses tumor growth in the MM mouse xenograft model. We further examined the underlying molecular mechanism and found that anlotinib provokes cell cycle arrest, induces apoptosis and inhibits multiple signaling pathways. Importantly, we identify c-Myc as a novel direct target of anlotinib. The enhanced ubiquitin proteasomal degradation of c-Myc contributes to the cell apoptosis induced by anlotinib. In addition, anlotinib also displays strong cytotoxicity against bortezomib-resistant MM cells. Our study demonstrates the extraordinary anti-MM effect of anlotinib both in vitro and in vivo, which provides solid evidence and a promising rationale for future clinical application of anlotinib in the treatment of human MM.
\end{abstract}

\section{Introduction}

Multiple myeloma (MM) is a hematologic malignancy characterized by the accumulation of clonal plasma cells predominantly in bone marrow ${ }^{1}$. Of all the hematologic malignancies, $\mathrm{MM}$ accounts for about $1.8 \%$, ranking third in the hematological malignant tumors after lymphoma and leukemia ${ }^{2}$. Over the last few decades, the advents of novel treatments, such as immunomodulatory drugs (e.g.,

\footnotetext{
Correspondence: Weiying Gu (guweiying2001@163.com) or

Yingli Wu (wuyingli@shsmu.edu.cn)

'Department of Hematology, The First People's Hospital of Changzhou, Third Affiliated Hospital of Soochow University, 213003 Changzhou, Jiangsu Province, P.R. China

${ }^{2}$ Hongqiao International Institute of Medicine, Shanghai Tongren Hospital/ Faculty of Basic Medicine, Chemical Biology Division of Shanghai Universities EInstitutes, Key Laboratory of Cell Differentiation and Apoptosis of the Chinese Ministry of Education, Shanghai Jiao Tong University School of Medicine, 200025 Shanghai, China

Full list of author information is available at the end of the article These authors contributed equally: Yang Cao, Huizhuang Shan, Meng Liu Edited by M. Diederich
}

lenalidomide, pomalidomide $)^{3}$, proteasome inhibitors (e.g., bortezomib, carfilzomib $)^{4}$, histone deacetylase inhibitors (e.g., panobinostat) ${ }^{5}$, monoclonal antibodies (e.g., daratumumab, elotuzumab) ${ }^{6}$, chimeric antigen receptor T-cell (CAR-T) therapy ${ }^{7}$, and autologous transplantation $^{8}$, have transformed the management for MM patients and improved the therapeutic outcomes. However, relapse or chemotherapy resistance is an expected part of the disease course, and MM heretofore has still been considered fatal and incurable 9 . Therefore, studies aiming to uncover new or alternative therapies are urgently needed to improve the clinical outcomes of MM.

Epidemiologic studies have demonstrated that symptomatic MM is consistently preceded by a precursor state named monoclonal gammopathy of undetermined significance (MGUS), followed by smoldering multiple myeloma $(\mathrm{SMM})^{10}$. Of note, unlike what is often present in MM patients, the translocations involving the $M Y C$ locus and the gains of $M Y C$ are rarely detected in patients

\section{(c) The Author(s) 2021}

(c) (i) Open Access This article is licensed under a Creative Commons Attribution 4.0 International License, which permits use, sharing, adaptation, distribution and reproduction cc) in any medium or format, as long as you give appropriate credit to the original author(s) and the source, provide a link to the Creative Commons license, and indicate if changes were made. The images or other third party material in this article are included in the article's Creative Commons license, unless indicated otherwise in a credit line to the material. If material is not included in the article's Creative Commons license and your intended use is not permitted by statutory regulation or exceeds the permitted use, you will need to obtain permission directly from the copyright holder. To view a copy of this license, visit http://creativecommons.org/licenses/by/4.0/. 
with MGUS and $\mathrm{SMM}^{11,12}$. In both the transition of MGUS to MM and the late stages of MM progression, cMyc is frequently activated and proposed to be a significant trigger ${ }^{13,14}$. c-Myc exerts neoplastic effects by integrating various signals to affect a diverse array of cellular processes, including DNA replication, cell growth, metabolism, cell cycle progression, and so on ${ }^{15-17}$. Since c-Myc dysregulation is a unique feature in the genetic landscape of $\mathrm{MM}$, it represents an attractive drug target for $M M$ treatment ${ }^{18}$. Until now, several strategies have been developed to target MYC in MM. However, many of these approaches are suffered from low potency and poor pharmacokinetic properties. Besides, direct inhibition of c-Myc in the area of ligand discovery remains a challenge.

Anlotinib (AL3818) is a novel oral multi-targeted receptor tyrosine kinase inhibitor (TKI) that targets vascular endothelial growth factor receptor (VEGFR) 1-3, c-Kit, plateletderived growth factor receptor (PDGFR) $-\alpha / \beta$, and fibroblast growth factor receptor (FGFR) 1-4. Anlotinib is considered as a broad-spectrum drug with inhibitory effects on tumor progression, apoptosis, proliferation, and angiogenesis ${ }^{19}$. Based on the result of phase 3 randomized clinical trial (ALTER-0303), anlotinib has become the first drug approved in China for a third-line treatment or beyond for advanced non-small cell lung carcinoma (NSCLC) ${ }^{20}$. Recent preclinical and ongoing clinical trials have demonstrated promising antitumor activities of anlotinib against diverse malignant tumor types (e.g., metastatic renal cell carcinoma ${ }^{21}$, advanced medullary thyroid carcinoma ${ }^{22}$, and soft tissue sarcoma ${ }^{23}$ ); however, no research has thrown light on its potential efficacy in MM.

This study is the first to report the therapeutic effects of anlotinib on MM and explore its possible molecular mechanisms. We found that anlotinib exerts potent antiMM activity in vitro and in vivo, regardless of the bone marrow microenvironment protection or bortezomibresistance. Further studies demonstrate that anlotinib directly interacts with c-Myc, accelerates its ubiquitin proteasome-dependent degradation, followed by genomewide downregulation of c-Myc-dependent target genes, which contributes to the anti-MM effect of anlotinib. Our work provides the proof-of-concept for clinical evaluation of anlotinib in the treatment of MM.

\section{Materials and methods}

\section{Cell culture and reagents}

The human MM cell lines NCI-H929, RPMI-8226, MM.1S, and U266 were purchased from the American Type Culture Collection (Manassas, VA, USA). LP1 and OPM2 were obtained from the German Collection of Microorganisms and Cell Cultures (Braunschweig, Germany). The cell lines were authenticated by short-tandem repeat profiling prior to use and were routinely tested negative for mycoplasma contamination. The bortezomib-resistant cells,
NCI-H929-BR, and MM.1S-BR, were induced by ongoing treatment of bortezomib. Bone marrow mononuclear cells (BMMCs) were isolated from three MM patients and four healthy donors using Ficoll-Hypaque density gradient centrifugation. $\mathrm{CD}_{138^{+}} \mathrm{MM}$ cells were purified from the BMMCs of MM patients by Human CD138 MicroBeads (Miltenyi Biotech, Auburn, CA). Bone marrow mesenchymal stem cells (BMSCs) were generated from BMMCs of MM patients. Informed consent was obtained in accordance with the Declaration of Helsinki protocol and approval by the Institutional Review Board of The Third Affiliated Hospital of Soochow University. All the cells were cultured in a humidified chamber at $37{ }^{\circ} \mathrm{C}$ in $5 \% \mathrm{CO}_{2}$ atmosphere, suspended in RPMI 1640 (MM cell lines) or DMEM medium (BMSCs), and added 10\% fetal bovine serum (SigmaAldrich, St.Louis, MO), $100 \mu \mathrm{g} / \mathrm{ml}$ streptomycin and $100 \mathrm{IU} /$ $\mathrm{ml}$ penicillin (Invitrogen).

Anlotinib, CHX and MG-132 were purchased from CSNpharm (Chicago, IL, USA), dissolved in Dimethyl Sulfoxide (Sigma, St. Louis, MO) and stored in dark at $-20{ }^{\circ} \mathrm{C}$ until use. Recombinant human interleukin 6 (IL-6) was obtained from R\&D Systems (Minneapolis, MN). Matrigel purchased from Corning Life Sciences (Corning, NY, USA).

\section{Cell viability assay}

The Cell Counting Kit-8 (CCK-8, Dojindo, Kumamoto, Japan) assay was used to measure the cell viability according to the manufacturer's instructions. Briefly, the cells were cultured in 96-well plates, treated with drugs at the indicated concentrations and then incubated with CCK- 8 working solution for $1-4 \mathrm{~h}$ at $37^{\circ} \mathrm{C}$. The resulting absorbance was detected at $450 \mathrm{~nm}$ in a microplate reader. The combined effect was assessed using the CompuSyn software (Biosoft, Ferguson, MO, USA). The combination index $(\mathrm{CI})<1,=1$, and $>1$ indicated synergism, addictive effect, and antagonism, respectively.

\section{Flow cytometric analysis for cell cycle and apoptosis}

For cell cycle distribution, cells were harvested, fixed with $70 \%$ ethanol overnight at $-20^{\circ} \mathrm{C}$ and incubated with $\mathrm{PI} / \mathrm{RNase}$ staining buffer (BD Biosciences) in the dark. The fluorescence of the stained cells was measured using a FACScan flow cytometer (Becton Dickinson, San Diego, CA, USA). Cell apoptosis was determined by flow cytometry after staining with Annexin V and PI detection kit (BD Biosciences, San Diego, CA, USA). Apoptotic cells include early (Annexin V positive and PI negative) and late (both Annexin V and PI positive) apoptosis.

\section{TUNEL assay and DAPI staining}

RPMI-8226 and NCI-H929 cells were treated with $5 \mu \mathrm{M}$ anlotinib for $24 \mathrm{~h}$. After fixed in $4 \%$ paraformaldehyde and permeabilized with $0.3 \%$ Triton X-100, cells were 
incubated with TUNEL detection solution $(50 \mu \mathrm{l} /$ well; Beyotime Institute of Biotechnology) for $60 \mathrm{~min}$ and then DAPI for $5 \mathrm{~min}$ at $37^{\circ} \mathrm{C}$. The cells were imaged under confocal microscopy (Nikon, Japan).

\section{Western blotting}

Cells were harvested, washed, and lysed. Equal quantities of protein extract were electrophoresed using sodium dodecyl sulfate-polyacrylamide gel electrophoresis and transferred to nitrocellulose membranes. After blocking with $5 \%$ non-fat milk, the membranes were incubated with primary antibodies overnight at $4{ }^{\circ} \mathrm{C}$, followed by incubation with the horseradish peroxidase (HRP)-conjugated secondary IgG antibody. The signals were visualized using the ECL detection system (Thermo Fisher, USA). Antibodies against PARP1 (\#9532), caspase 3 (\#9662) and 9 (\#9502), pERK1/2 (T202/Y204, \#4370), ERK1/2 (\#4695), p-AKT (S473, \#4060), AKT (\#4691), p-mTOR (S2448, \#5536), mTOR (\#2983), p-STAT3 (Y705, \#9145), STAT3 (\#4904), p-p65 (S536, \#3033), p65 (\#8242), MAX (\#4239), and $\beta$-actin (\#4970) were purchased from Cell Signaling Technology (Beverly, MA, USA); c-Myc (\#ab32072), p-c-Myc (T58, \#ab28842), and p-c-Myc (S62, \#ab51156) was from Abcam (Cambridge, UK).

\section{Quantitative reverse transcription real-time PCR (qRT-PCR)}

Total RNA of the agent-treated cells was extracted using TRIzol reagent (Invitrogen). Thereafter, the cDNA was synthesized using a cDNA reverse transcription kit (TransGen Biotech, Beijing, China). The qRT-PCR was performed in triplicate on $7900 \mathrm{HT}$ real-time PCR instrument (Applied Biosystems, CA, USA) using the SYBR Green Mix (Applied Biosystems). The primer sequences of c-Myc were as follows: forward primer, CTGCGTAGTTGTGCTGATGT; reverse primer, ATC ATTTCCATGACGGCCTGT. The relative expression level of c-Myc was normalized to $\beta$-actin and then presented by the $2^{-\Delta \Delta C t}$.

\section{Mouse xenograft model}

The BALB/c nude mice (5-weeks old) were purchased from the Shanghai Laboratory Animal Center (Shanghai, China). The animal experiments were approved by the Animal Research Committee of The Third Affiliated Hospital of Soochow University and were performed in accordance with established guidelines. Mice were bred in the specific pathogen-free (SPF) grade of the animal care facility and a $12 \mathrm{~h}$ dark-light cycle. After acclimatization, $1 \times 10^{7}$ NCI-H929 cells were mixed with Matrigel and subcutaneously injected into the right flank of the mice. When tumors were measurable, mice were randomly divided into treatment and control groups $(n=6$ mice each). In the treatment group, mice received $3 \mathrm{mg} / \mathrm{kg}$ anlotinib orally daily for consecutive 14 days. Tumor size and mouse body weight were monitored every other day. Tumor volume $\left(\mathrm{mm}^{3}\right)$ was calculated as length $\times$ width $^{2} / 2$. After 14 days of treatment, all the mice were killed, and the tumor tissues were weighed and prepared for western blotting and immunohistochemistry (IHC).

\section{Lentiviral packaging}

Lentiviral vectors were co-transfected with packaging plasmids psPAX2 and pMD2G into HEK293T cells. Infectious lentiviruses were harvested and filtered through $0.45 \mu \mathrm{m}$ PVDF filters. Recombinant lentiviruses were concentrated 100-fold by ultracentrifugation $(2 \mathrm{~h}$ at $120,000 \times g)$. MM cells were prepared and infected by lentiviruses, and western blotting was used to validate the expression of c-Myc. The shRNA sequences of c-Myc were: sh-1\#: 5'-CCTGAGACAGATCAGCAACAA-3' and sh-2\#: 5'-CCTGAGACAGATCAGCAACAA-3'.

\section{RNA sequencing (RNA-seq)}

Total RNA was extracted from the NCI-H929 cells after treated DMSO or anlotinib for $12 \mathrm{~h}$ using Trizol Reagent (Invitrogen, USA). Then, the RNA samples were sent to Gminix, Biotechnology Co, Ltd (Shanghai, China) for RNA-seq on an Illumina HiSeq $\times 10$ sequencing platform with $10 \mathrm{G}$ reads. The procedures were performed as described in detail on the website of Gminix. The raw data for this study is available at the NCBI sequence read archive (SRA) database under the accession number: PRJNA587019.

\section{Preparation of recombinant wild-type c-Myc protein}

Human full-length c-Myc were cloned into a pET-30c vector containing a $6 \times$ His tag at the C-terminal region. The E. coli strain BL21 was transformed with pET-c-Myc, cultured in $2 \times \mathrm{YT}$ and induced with $0.2 \mathrm{mM}$ IPTG for $6 \mathrm{~h}$. After centrifugation, the supernatant was applied to a $\mathrm{Ni}$ beads column and washed with wash buffer. Proteins were eluted with elution buffer.

\section{Cellular thermal shift assay (CETSA)}

CETSA is a newly developed approach based on the thermal stabilization of ligand-bound proteins. The procedure of CETSA has been described previously ${ }^{24}$. Briefly, cell lysates from NCI-H929 cells or purified c-Myc proteins were incubated with anlotinib or DMSO for $30 \mathrm{~min}$, and then heated at different temperatures for $3 \mathrm{~min}$. The mixtures were centrifuged, and the supernatants were subjected to western blotting.

\section{Drug affinity responsive targets stability (DARTS)}

DARTS was conducted in NCI-H929 cells following the previously published protocol ${ }^{25}$. Briefly, the cell lysates were prepared with lysis buffer and the concentration was determined as described above. The cell lysates were 
incubated with anlotinib or DMSO for $1 \mathrm{~h}$ at room temperature, and then digested with Pronase (Roche) for an additional $20 \mathrm{~min}$ at room temperature. The reaction was quenched by the protein inhibitor cocktail and the samples were subjected to western blotting.

\section{Co-immunoprecipitation (Co-IP)}

Co-IP was conducted to detect the protein interaction. Briefly, whole cell lysates were incubated with indicated antibodies and Protein G/A beads overnight at $4{ }^{\circ} \mathrm{C}$. After four times wash with RIPA buffer, beads were boiled in loading buffer to elute protein complexes, followed by western blotting with specific antibodies. HRP-conjugated AffiniPure Mouse Anti-Rabbit IgG Light Chain was purchased from ABclonal (China, \#AS061).

\section{Statistical analysis}

Statistical analysis was performed using SPSS version 22.0 software (IBM Corporation, Chicago). The quantitative data are shown as the mean $\pm \mathrm{SD}$. The data were examined using the two-tailed Student's $t$-test or one-way ANOVA with post-hoc Bonferroni correction. The $\mathrm{IC}_{50}$ value was calculated based on the dose-response curve using GraphPad Prism 8.0 software. Statistical significance is indicated by ${ }^{*} P<0.05,{ }^{* *} P<0.01$, and ${ }^{* * * *} P<0.001$.

\section{Results}

Anlotinib exhibits potent cytotoxicity against MM cell lines and primary cells

To determine whether anlotinib (Fig. 1A) has a cytotoxic effect on human MM cells, we conducted CCK-8

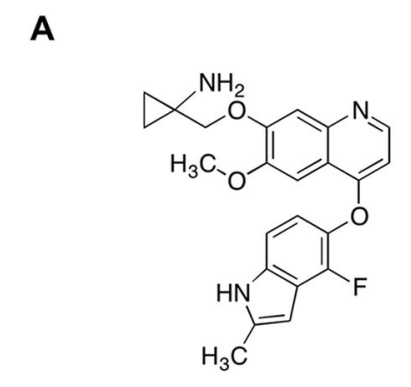

C

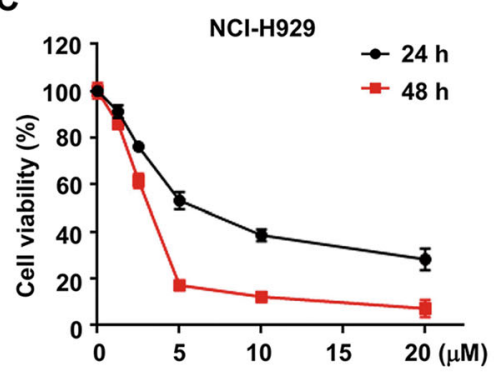

$\mathbf{F}$

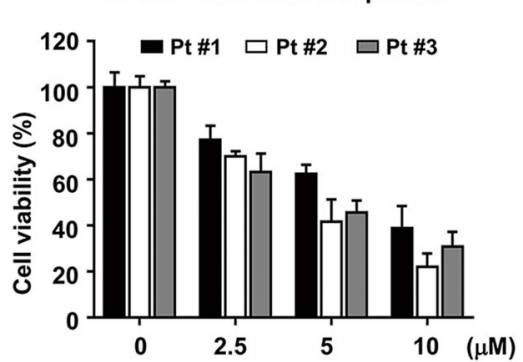

B

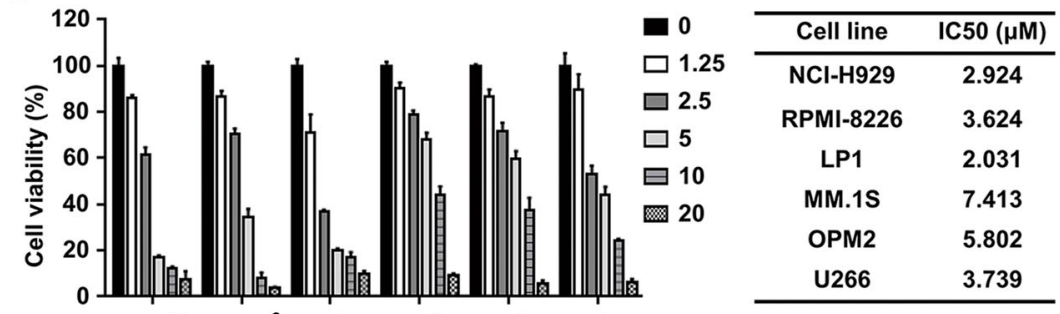

D

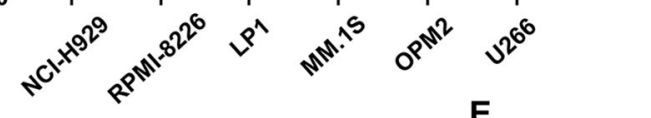

E
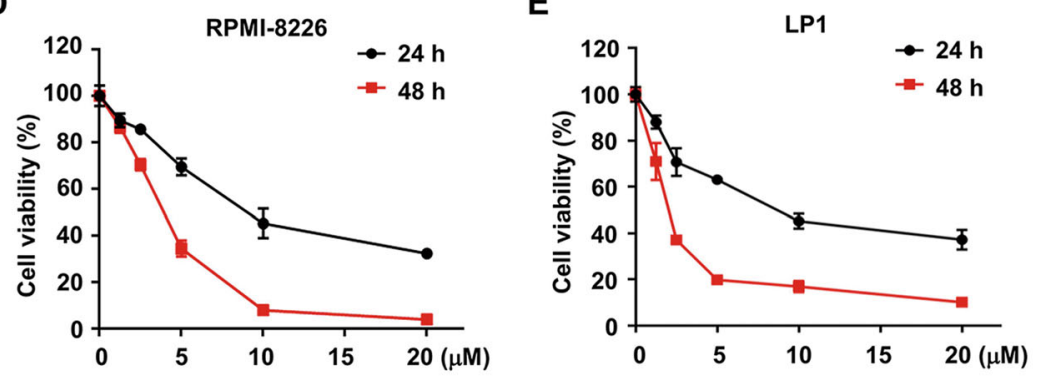

H

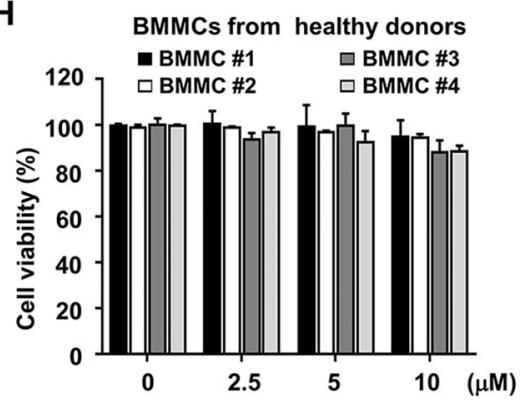

Fig. 1 Anlotinib exerts potent cytotoxicity against MM cells. A Chemical structure of anlotinib. B MM cell lines (NCI-H929, RPMI-8226, LP1, MM.1S, OPM2, and U266) were treated with anlotinib (0-20 $\mu \mathrm{M})$ for $48 \mathrm{~h}$, and the cell viability was detected by CCK8. The IC50 value was calculated based on the dose-response curve using Prism 8.0 software. C-E NCI-H929, RPMI-8226 and LP1 cells were treated with anlotinib for 24 and 48 h, followed by

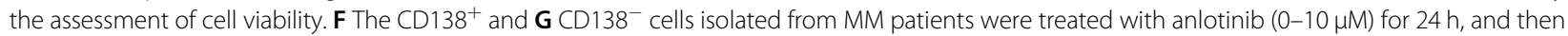
the cell viability was assessed. $\mathbf{H}$ Normal BMMCs from four healthy donors were treated with anlotinib $(0-10 \mu \mathrm{M})$ for $24 \mathrm{~h}$. Data are shown as mean \pm SD and representative of three independent experiments. 
A

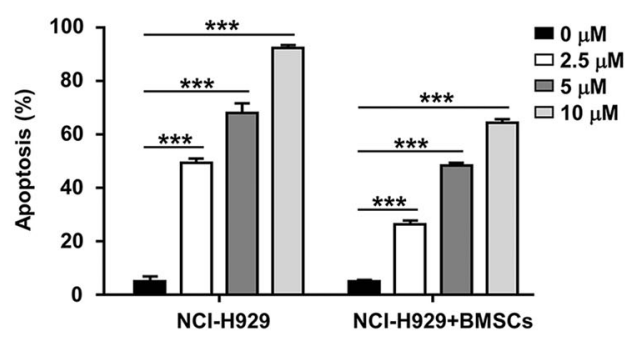

C

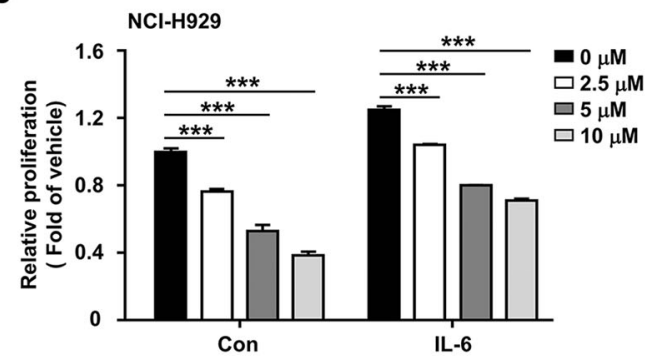

B

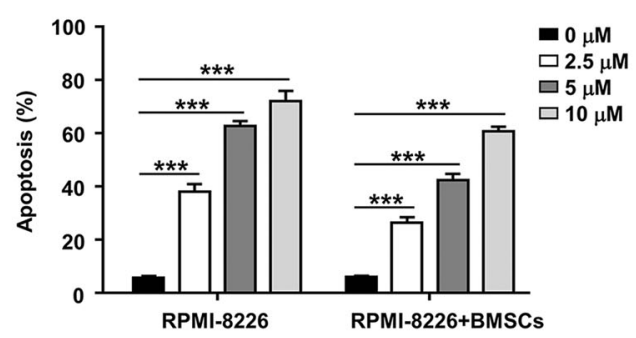

D

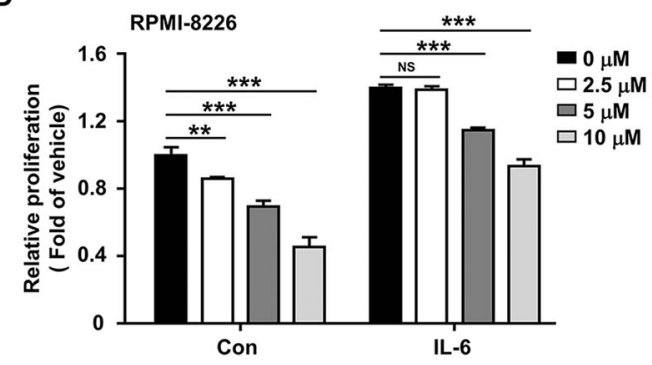

Fig. 2 Anlotinib overcomes the protective effect of the BMSCs and exogenous IL-6 on MM cells. A, B NCI-H929 and RPMI-8226 cells were COcultured with or without BMSCs, and then treated with anlotinib $(0-10 \mu \mathrm{M})$ for $48 \mathrm{~h}$. After staining with Annexin $\mathrm{V}$ and PI, flow cytometry analysis was performed to assess the apoptosis rate. C, D NCl-H929 and RPMI-8226 cells were treated with anlotinib $(0-10 \mu \mathrm{M})$ for $48 \mathrm{~h}$, in the presence or absence of IL-6 $(10 \mathrm{ng} / \mathrm{ml})$. Cell proliferation was measured by CCK-8 assay. ${ }^{* *} P<0.01 ;{ }^{* * *} P<0.001$; ${ }^{\mathrm{NS}} P>0.05$. Data are shown as mean \pm SD and from three independent experiments.

assay in a panel of MM cell lines (NCI-H929, RPMI-8226, LP1, MM.1S, OPM2, and U266). As shown in Figs. 1B and S1, anlotinib-induced dose-dependent cytotoxicity in all MM cell lines with $\mathrm{IC}_{50}$ values ranging from 2.0 to 7.4 $\mu \mathrm{M}$. We also treated NCI-H929, RPMI-8226, and LP1 cells with anlotinib $(0-20 \mu \mathrm{M})$ for 24 and $48 \mathrm{~h}$, and observed that the cell viabilities were inhibited in a timedependent manner (Fig. 1C-E). Moreover, the anti-MM effect of anlotinib was also observed in $\mathrm{CD} 138^{+} \mathrm{MM}$ cells isolated from BMMCs of three MM patients (Fig. 1F). In contrast, anlotinib had a low or no cytotoxic effect on CD138 ${ }^{-}$cells from MM patients or BMMCs from healthy donors (Fig. 1G, H). These findings indicated that anlotinib has potent cytotoxicity in MM cell lines and primary cells, but has a faint effect on normal cells.

\section{Anlotinib antagonizes the protective effect of bone marrow microenvironment on MM cells}

Given that the bone marrow microenvironment promotes the proliferation, survival, and chemoresistance of MM cells, we explored whether anlotinib could overcome the protective effect of bone marrow microenvironment. First, we detected the cytotoxicity of anlotinib on NCIH929 and RPMI-8226 cells when co-cultured with patient-derived BMSCs. Notably, anlotinib induced remarkable apoptosis of MM cells even in the presence of BMSCs (Fig. 2A, B), while had no significant cytotoxicity in BMSCs (Fig. S2). In addition, the bone marrow microenvironment was mimicked by culturing $\mathrm{MM}$ cells in the presence of IL-6. As shown in Fig. 2C, D, although IL-6 effectively promoted the proliferation of MM cells, anlotinib still induced cytotoxicity in both NCI-H929 and RPMI-8226 cells. These data suggested that anlotinib not only directly targets $\mathrm{MM}$ cells, but also partially overcomes the proliferative and anti-apoptotic effects mediated by the MM-host bone marrow microenvironment.

\section{Anlotinib induces cell cycle arrest and apoptosis in MM cells}

Since cell growth is closely related to cell cycle progression, we evaluated the cell cycle distribution of NCIH929 and RPMI-8226 cells treated with anlotinib. The results showed that anlotinib caused a significant accumulation of cells in the $G_{2} / M$ phase (Fig. 3A). The morphological staining revealed increased cell fragments and debris in anlotinib-treated MM cells, which indicated the potential impact of anlotinib on cell apoptosis (Fig. 3B). Nuclear morphology was assessed by DAPI staining, and showed the formation of the dot-like apoptotic body in anlotinib-treated cells, while the nucleus of control cells was round in shape (Fig. 3C). In addition, TUNELpositive cells increased strikingly in NCI-H929 and RPMI8226 cells after anlotinib treatment (Fig. 3C). Consistent with these findings, anlotinib induced cleavage of caspase 3 and 9, as well as PARP-1 (Fig. 3D). As assessed by flow cytometry, anlotinib treatment resulted in a dose- 


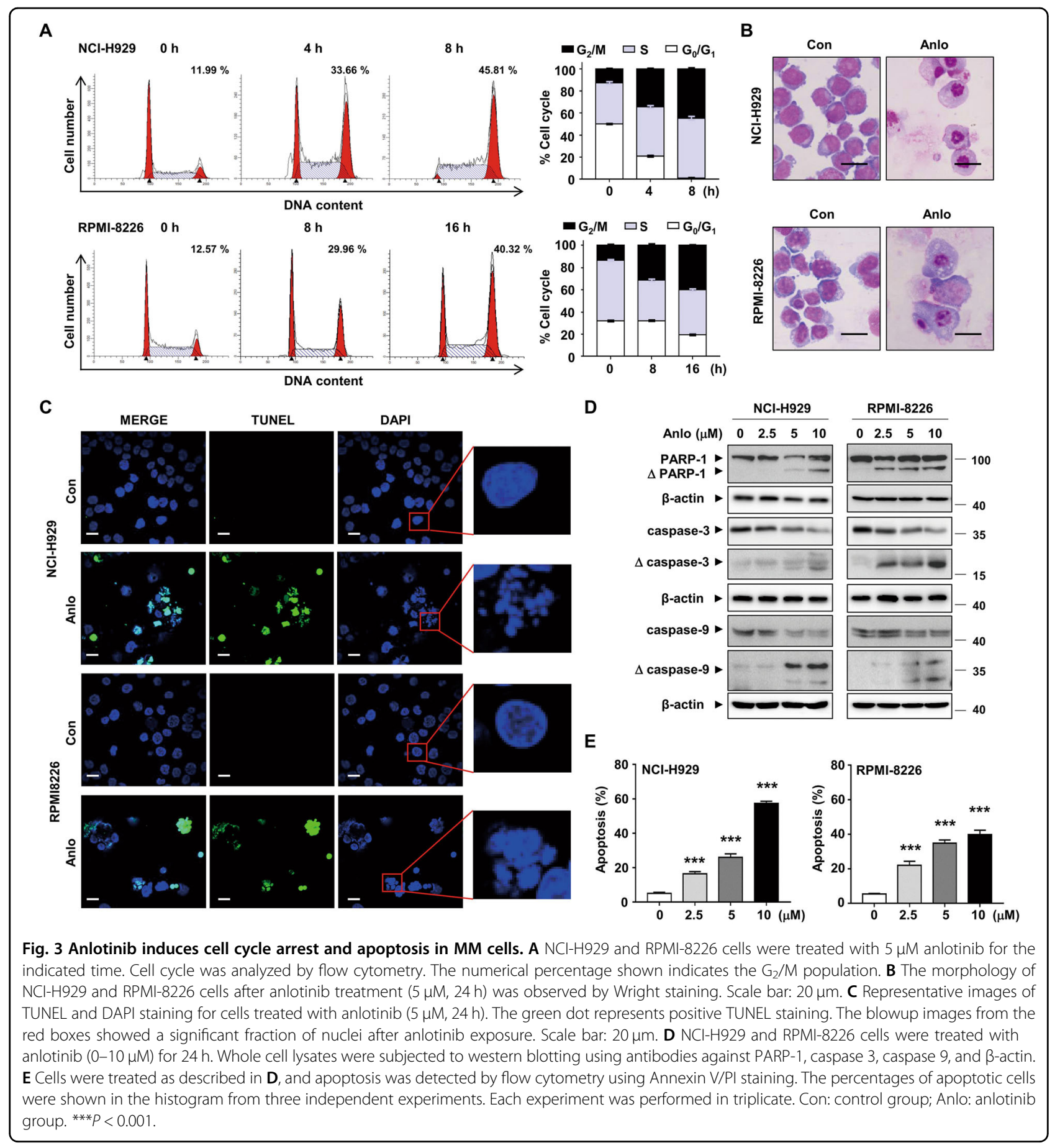

dependent increase in Annexin V-positive MM cells (Fig. 3E). Thus, these data demonstrated that anlotinib induces cell cycle arrest and apoptosis of MM cells.

Genome-wide transcriptome analysis indicates c-Myc as a key regulator of anlotinib-treated MM cells

Based on the observations of anti-MM effects exerted by anlotinib, we employed RNA-Seq technology to explore the underlying molecular mechanisms. A total of 842 differentially expressed genes (DEGs) were identified (Fig. 4A) and subjected to Kyoto Encyclopedia of Genes and Genomes (KEGG) analysis. The results revealed that the DEGs were mainly involved in the multiple signaling pathways which are important for MM cell proliferation and survival (Fig. 4B). Consistent with the results of RNASeq, we observed inhibited MAPK, PI3K/Akt/mTOR, 


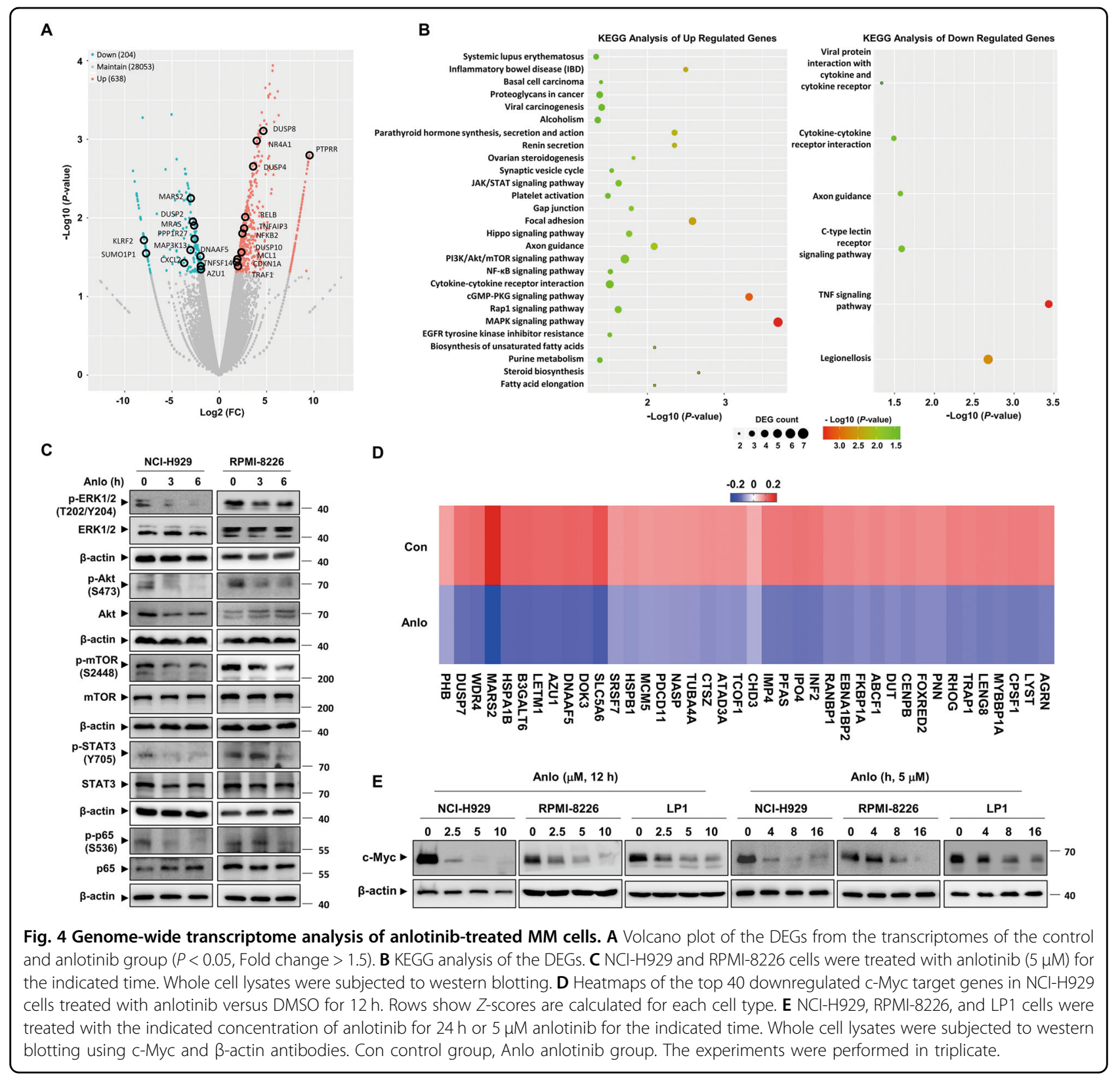

JAK/STAT, and NF- $\mathrm{BB}$ signaling in anlotinib-treated NCI-H929 and RPMI-8226 cells (Fig. 4C). It is widely recognized that oncogene c-Myc has a vital role in the pathophysiology of MM and is regulated by a large network of interconnected signaling pathways involves MAPK, PI3K/Akt, JAK/STAT, and NF-кB ${ }^{26-29}$. Intriguingly, further analysis of the DEGs heatmap showed that the genes associated with the c-Myc targets were markedly downregulated by anlotinib treatment (Fig. 4D). Then, we examined the levels of c-Myc protein in three MM cell lines after anlotinib treatment, and the results showed that anlotinib dramatically down-regulated c-Myc in a dose- and time-dependent manner without effect on its mRNA levels (Figs. 4E and S3). Thus, these results indicated that anlotinib reduces $\mathrm{c}-\mathrm{Myc}$ protein probably at the post-transcriptional level.

\section{Anlotinib directly targets c-Myc and promotes its ubiquitin proteasomal degradation}

To measure the half-life of c-Myc in the presence of anlotinib, NCI-H929 cells were co-treated with cycloheximide (CHX) to block de novo protein synthesis. The result showed that anlotinib reduced the half-life of c-Myc protein by 2 -fold (Fig. 5A). The increased turnover of c-Myc protein after anlotinib treatment could be rescued by proteasome inhibitor MG-132, suggesting that the destruction 


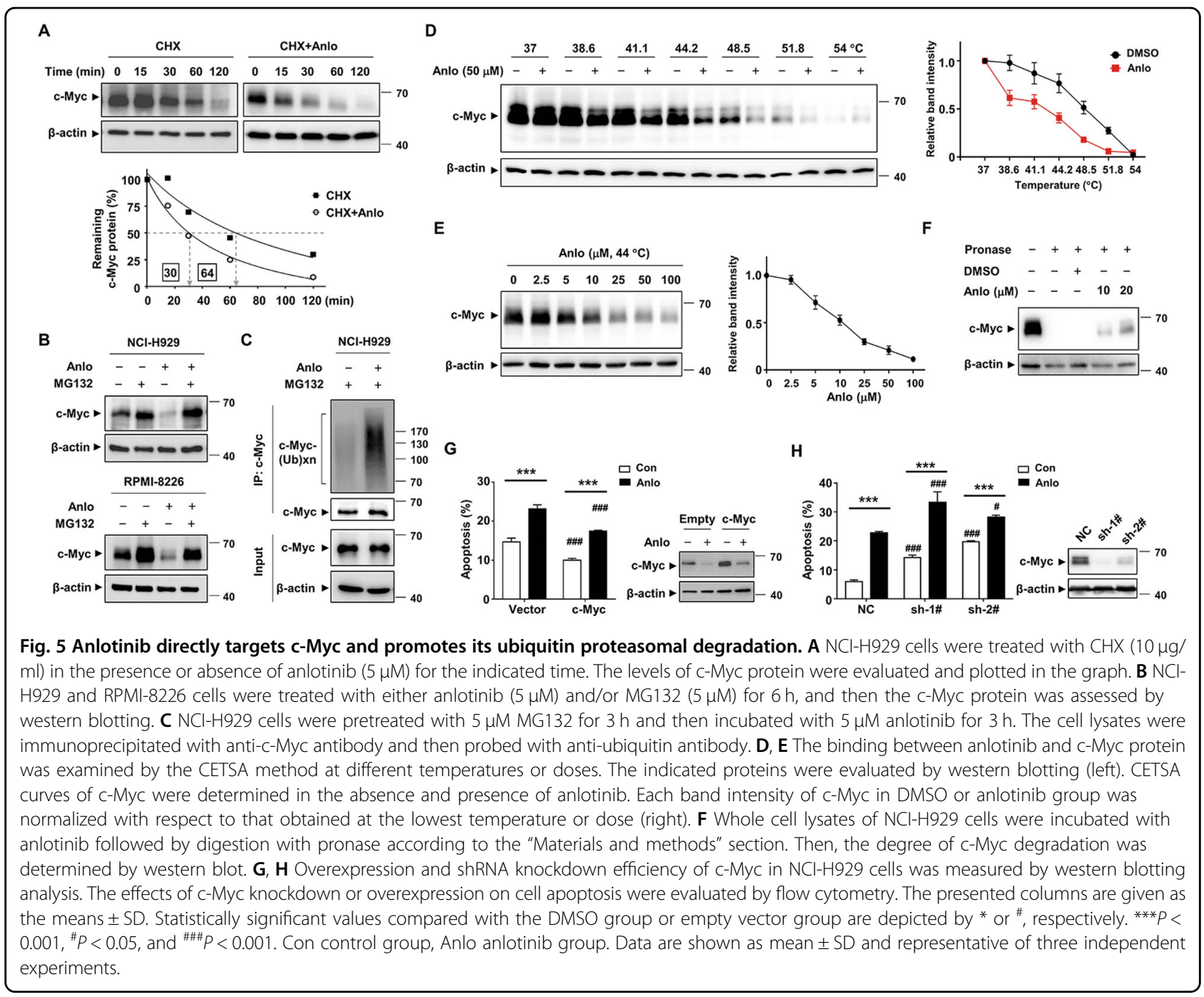

of c-Myc is mediated by the ubiquitin-proteasome system (Fig. 5B). We found that the level of ubiquitinated c-Myc species increased upon anlotinib treatment (Fig. 5C). In addition, anlotinib triggered the dephosphorylation of cMyc at S62 and phosphorylation at T58 (Fig. S4). Whereas, anlotinib did not affect the interaction between c-Myc and its partner protein Max (Fig. S5). To clarify the molecular basis for the inhibition of c-Myc induced by anlotinib, we evaluated the possibility of c-Myc protein as a cellular direct target of anlotinib. The results of CETSA showed that the thermal stability of c-Myc protein was tapered by anlotinib in a temperature-dependent and dose-dependent manner (Figs. 5D, E and S6). To further confirm the interaction between anlotinib and c-Myc, DARTS technology was performed. As expected, anlotinib significantly protected c-Myc protein from digestion by pronase, indicating that anlotinib interacts directly with c-Myc in vitro (Fig. 5F). Moreover, we found that forced overexpression of c-Myc in NCI-H929 by lentiviral infection rescued, in part, the apoptosis induced by anlotinib (Fig. 5G). Conversely, the shRNA silencing of $\mathrm{c}-\mathrm{Myc}$ increased the apoptosis rate (Fig. 5H). Whereas, neither overexpression nor knockdown of c-Myc caused significant changes in the cell cycle distributions (Fig. S7). Overall, these results indicated that anlotinib directly interacts with $\mathrm{c}-\mathrm{Myc}$ and promotes its ubiquitin proteasomal degradation, which partially contributes to the apoptosis induced by anlotinib.

\section{Anlotinib is cytotoxic to bortezomib-resistant MM cells}

To examine the cytocidal effects of anlotinib on bortezomib-resistant cells, we established NCI-H929-BR and MM.1S-BR cell lines which are effectively shown resistant to bortezomib (Fig. 6A). As showed in Fig. 6B, anlotinib remarkably suppressed the viability of NCIH929-BR and MM.1S-BR in a dose-dependent and timedependent manner. Similar to the bortezomib-sensitive cells, the induction of $\mathrm{G}_{2} / \mathrm{M}$ arrest and apoptosis was also detected in NCI-H929-BR and MM.1S-BR cells after 


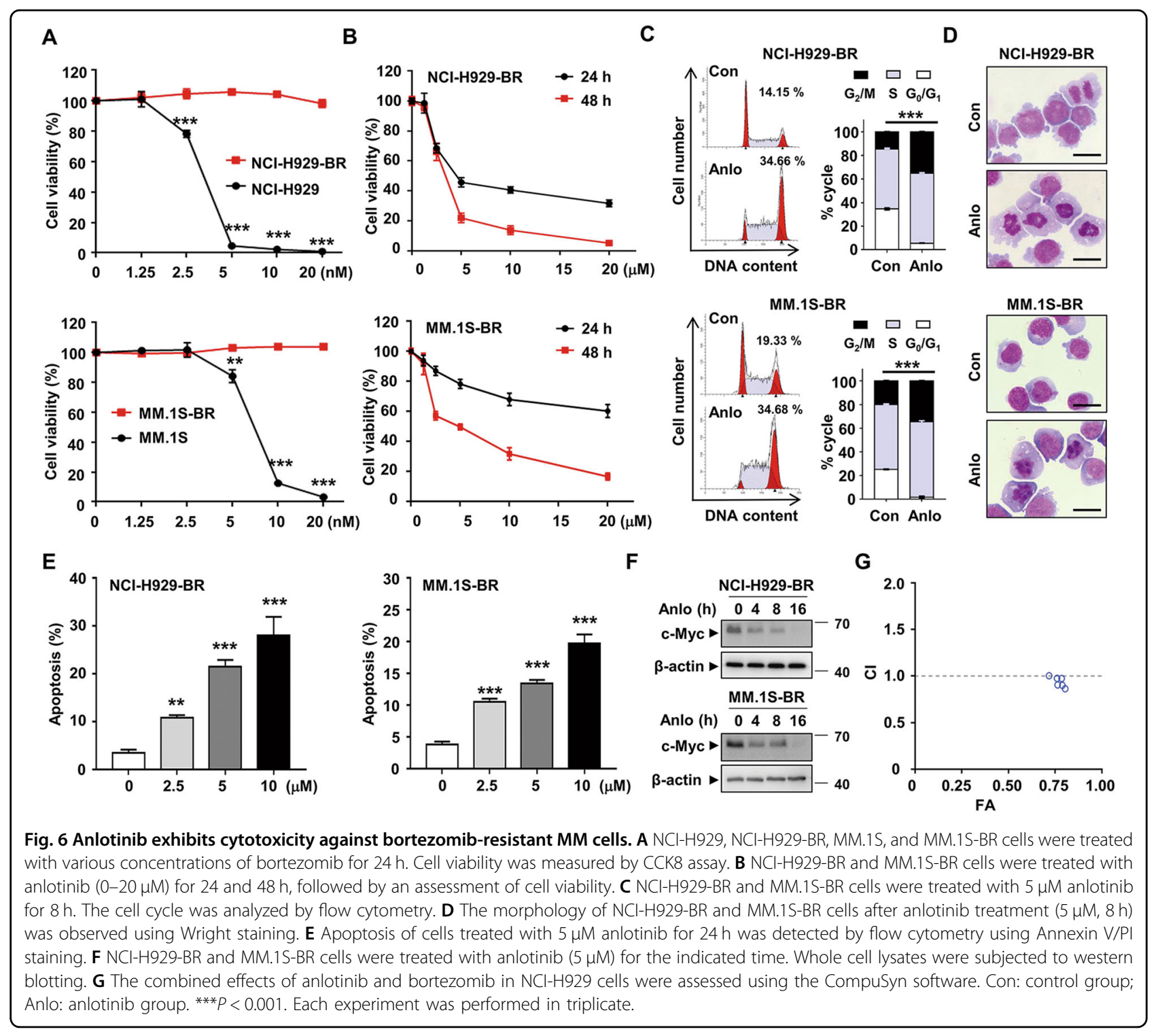

exposure to anlotinib (Fig. 6C-E). We also analyzed the expression change of c-Myc protein and obtained similar results as shown in Fig. 6F. In addition, the $\mathrm{CI}$ values of anlotinib and bortezomib were nearly 1 , which indicates an additive effect (Fig. 6G and Supplementary Table 1). Taken together, these data suggested that anlotinib exerts strong cytotoxicity against bortezomib-resistant MM cells.

\section{Antitumor efficacy of anlotinib in a MM xenograft model in vivo}

Considering the encouraging anti-MM efficacy of anlotinib in vitro, we next evaluated the in vivo therapeutic potential of anlotinib in MM xenograft model. Six-weekold nude mice bearing subcutaneous NCI-H929 cells were treated with either anlotinib or vehicle control by intragastric administration for consecutive 14 days. Tumor growth and weight in the anlotinib-treated group were dramatically inhibited by approximately $69.3 \%$ and $77.2 \%$, compared with the vehicle-treated group (Fig. 7A-C). No significant body weight loss (Fig. 7D) and treatmentrelated death were observed, indicating that anlotinib was well-tolerated. As shown in Fig. 7E, both the cell proliferation and vascular density were significantly suppressed, demonstrated by reduced Ki-67 staining and decreased CD31-positive microvessels, respectively. The activation of apoptosis induced by anlotinib was revealed by the increase of cleaved-caspase 3 and TUNEL-positive cells. Moreover, the IHC and western blotting analyses of the harvested tumor tissues showed that anlotinib markedly inhibited c-Myc expression in vivo (Fig. 7E, F). Taken together, these findings suggested that anlotinib has potent anti-MM activity without additional toxicity in vivo. 


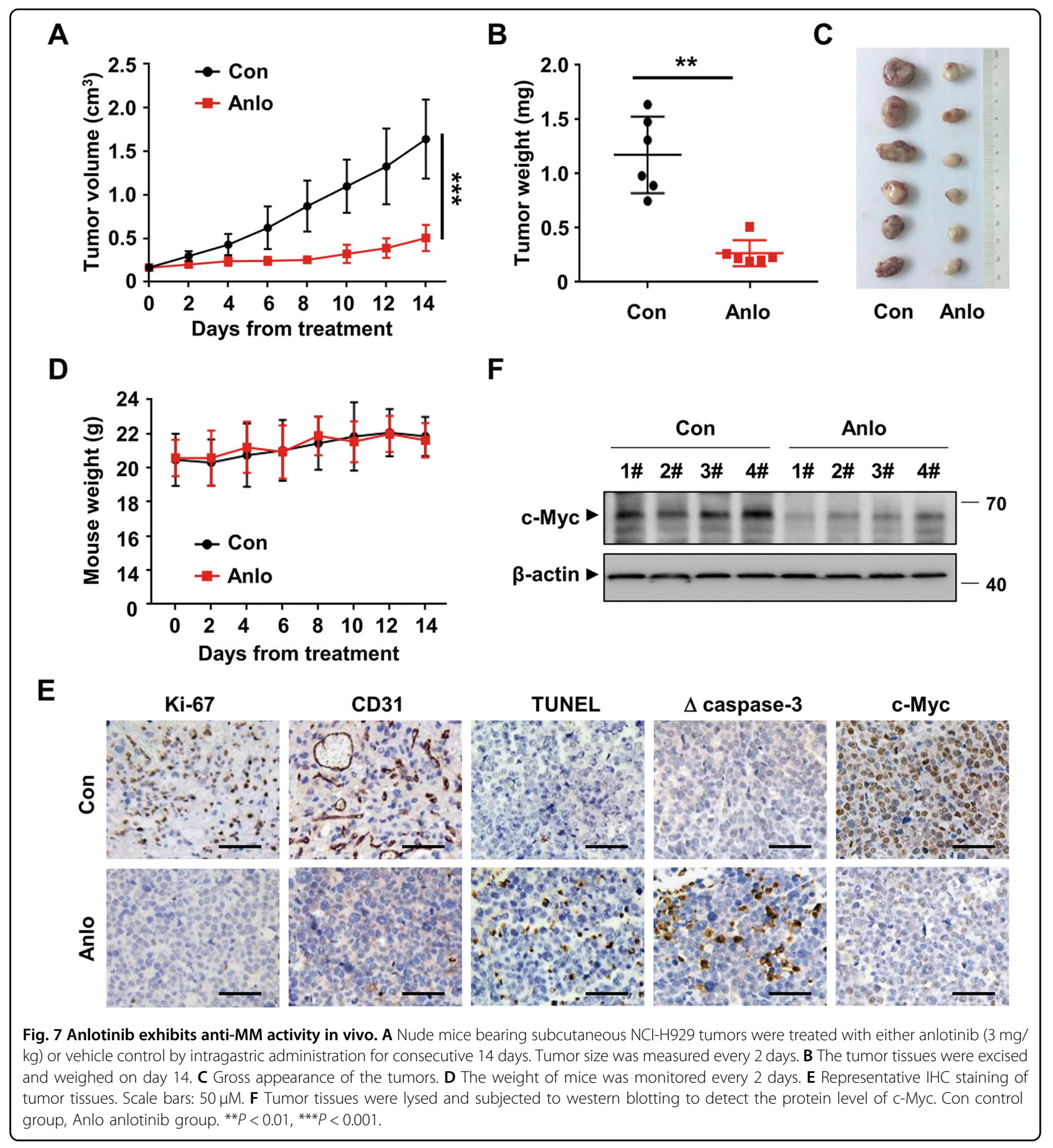

\section{Discussion}

In the present study, we found that anlotinib is an exciting and promising therapeutic agent to treat MM both in vitro and in vivo. Unlike what has been described as the targets of anlotinib (e.g., VEGFR 1-3, c-Kit, PDGFR- $\alpha / \beta$, and FGFR $1-4)^{20}$, we identify c-Myc for the first time as a novel direct target of anlotinib. Anlotinib displays strong cytotoxicity against bortezomib-sensitive and bortezomib-resistant MM cells, overcomes the protective effect of the bone marrow microenvironment and suppresses tumor growth in the MM mouse xenograft. Our work demonstrates the extraordinary anti-MM effect of anlotinib, which may provide a promising therapeutic strategy for human MM.

Similar to other oncogenic transcription factors, c-Myc has long been viewed as an undruggable therapeutic 
target due to the lack of kinase activity and intrinsically disordered structure. Several strategies have been developed to indirectly target c-Myc including blocking Myc transcription $^{30,31}$, inhibiting bromodomain proteins ${ }^{32}$ and down-regulating downstream genes of $\mathrm{MYC}^{33,34}$. Nonetheless, the most reliable strategy is to target c-Myc directly, which abrogates downstream MYC oncogenic activities regardless of upstream alterations. Recently, small molecules that directly disrupt the c-Myc/Max interaction and DNA binding are attracting much attention $^{35-37}$. However, many of these inhibitors are suffered from low potency and poor pharmacokinetic properties. One interesting finding in our study is the identification of c-Myc as the novel target of anlotinib, which was supported by several lines of evidence. First, the genome-wide transcriptome analysis revealed a series of downregulated target genes regulated by c-Myc, indicating c-Myc as a potential regulator in anlotinib-treated MM cells. Second, anlotinib markedly inhibited c-Myc expression in MM cells without effect on its mRNA levels. Third, the CETSA and DARTS assay confirmed the direct interaction between anlotinib and c-Myc. In 2013, Pär Nordlund et al. introduced CETSA which is a well-established assay for measurements of physical interactions between the protein and a ligand. It is based on the principle that heat could induce the unfolding and precipitation of proteins. Pär Nordlund proposed that the protein stability will increase in presence of the ligand. In our opinion, the thermal stability of proteins upon ligand binding may decrease as well, which depends on whether the binding state between ligand and protein promotes or hinders the unfolding and precipitation of the protein. The destabilization of the protein upon ligand binding has also been reported in several literatures ${ }^{38,39}$. DARTS is a label-free method for target identification of bioactive small molecules, which is based on the concept that the protease susceptibility of the target protein will decrease upon drug binding ${ }^{25}$. DARTS result demonstrated that anlotinib could directly bind to c-Myc, rendering it to be resistant to pronase treatment probably because of conformational changes induced by tight binding of anlotinib. More intriguingly, anlotinib triggered the S62 dephosphorylation and T58 phosphorylation of c-Myc, and promoted the ubiquitin proteasome-mediated degradation of c-Myc, which partially contributes to anlotinibinduced apoptosis in MM cells. Although we cannot fully clarify why c-Myc protein degradation occurs after anlotinib binding, it is possible that c-Myc protein conformation is changed upon anlotinib binding, and thus increasing its association with E3 ubiquitin ligases or decreasing its association with deubiquitinases, which subsequently enhances the proteasome-dependent degradation of c-Myc. In addition to MM, c-Myc is overexpressed and activated in many other cancers.
Interestingly, we have investigated the effects of anlotinib on other cancer cell lines (HL60, SU-DHL-2, and OCILy3) with strong c-Myc dependency. The results showed decreased cell viability of these cells with down-regulation of c-Myc expression after anlotinib treatment, which are similar to the effect of anlotinib in MM cells (Fig. S8). Recent preclinical and ongoing clinical trials have demonstrated promising anti-tumor activities of anlotinib against diverse malignant tumors ${ }^{21-23}$. More studies are needed to investigate whether the degradation of c-Myc contributes to the effects of anlotinib or the expression of c-Myc affects the sensitivity of anlotinib.

Additionally, we found that overexpression of c-Myc just partially rescued the apoptosis induced by anlotinib in $M M$, indicating there are other mechanisms. Although MM lacks disease-defining tyrosine kinase fusion genes, it is characterized by the activation of multiple tyrosine kinase signaling cascades. The tumor-specific driver aberrations of MM, such as genetic abnormalities and microenvironment-driven deregulations, promote disease progression partly mediated by signaling molecules. The signaling molecules include IL- $6{ }^{40}$, transforming growth factor-beta (TGF- $\beta)^{41}$, VEGF $^{42}$, insulin-like growth factor

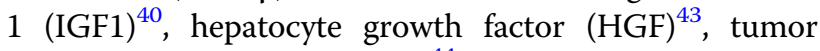
necrosis factor-alpha (TNF- $\alpha)^{44}$, Fms-like tyrosine kinase 3 (FLT3 $)^{45}$, and so on. These cytokines function upstream of RTKs and regulate multiple downstream signaling cascades including the Ras/Raf/MEK/MAPK, PI3K/Akt/ mTOR, JAK/STAT, and NF-kB pathway. Thus, targeting the activated RTKs signaling cascades with TKIs may be a promising therapeutic strategy against MM. Currently, numerous TKIs are being studied in MM, especially in the relapsed/refractory setting ${ }^{46}$. Nevertheless, so far, no TKIs have been approved for MM treatment, indicating the need for preclinical research to identify novel compounds. The well-recognized molecular targets for anlotinib are VEGFR1-3, c-Kit, PDGFR- $\alpha / \beta$, and FGFR1-4, the majority of which are abnormally active in $\mathrm{MM}^{47-49}$. Considering the core downstream pathways that RTKs signaling cascades converge at, we investigated the effects of anlotinib on those pathways and found that anlotinib inhibits the MAPK, PI3K/Akt/mTOR, JAK/STAT, and NF- $\mathrm{KB}$ pathways in MM cells. Moreover, accumulating evidence indicates that bone marrow microenvironment mediates drug resistance by offering protection against cytotoxic agents, leading to the selection and outgrowth of malignant cells under the selective pressure of drug exposure $^{50,51}$. Our results show that anlotinib antagonizes the protective effect of the bone marrow microenvironment and effectively induced cytotoxicity against bortezomib-resistant MM cells.

In summary, anlotinib exerts promising anti-MM activity in cultured and primary MM cells, as well as in MM mouse xenograft model. Directly targeted degradation of c-Myc is 
a novel mechanism by which anlotinib causes apoptosis in MM cells. Moreover, anlotinib could effectively induce cytotoxicity against bortezomib-sensitive and bortezomibresistant MM cells, and antagonize the protective effect of the bone marrow microenvironment. These results provide a basis for future clinical trials to investigate the efficacy and safety of anlotinib in the treatment of MM.

\section{Author details \\ 'Department of Hematology, The First People's Hospital of Changzhou, Third Affiliated Hospital of Soochow University, 213003 Changzhou, Jiangsu Province, P.R. China. ${ }^{2}$ Hongqiao International Institute of Medicine, Shanghai Tongren Hospital/Faculty of Basic Medicine, Chemical Biology Division of Shanghai Universities E-Institutes, Key Laboratory of Cell Differentiation and Apoptosis of the Chinese Ministry of Education, Shanghai Jiao Tong University School of Medicine, 200025 Shanghai, China. ${ }^{3}$ Department of Hematology, Ruijin Hospital, Shanghai Jiao Tong University School of Medicine, 200025 Shanghai, China}

\section{Author contributions}

Y.C., Y.-L.W. and W.-Y.G. designed the project and experiments. Y.C., H.-Z.S., M.L., Z.-L.Z., J.L., X.-G.X., Y.L. and H.-Z.X. conducted the experiments. Y.C., H.L., M.Y., X.M.Z., W.-T.L., B.-Z.L., F.-Z.X., Y.-J.J. and H.Y. analyzed the data and generated the figures. Y.C. and Y.-L.W. wrote the manuscript, and Y.-L.W. and W.-Y.G. supervised all aspects of the work. All authors involved in the review and revision of the manuscript, and approved the final manuscript.

\section{Funding}

This work was supported partly by Funding from Young Talent Development Plan of Changzhou Health Commission (CZQM2020023), The National Natural Science Foundation Youths of China (No. 81700157), The Science and Technology Bureau of Changzhou (CJ20200118), National Key Research and Development Program of China (No. 2017YFA0505200), Science and Technology Committee of Shanghai (19ZR1428700), Innovative research team of high-level local universities in Shanghai and Foundation of 333 Project of Jiangsu Province, China (BRA2018014).

\section{Data availability}

The data that support the findings of this study are available from the corresponding author upon reasonable request.

\section{Ethical approval}

Based on the declaration of Helsinki, this study was approved by the Ethics Committee of The Third Affiliated Hospital of Soochow University, The First People's Hospital of Changzhou. Written informed consent was obtained before treatments, which are not publicly available since the database is not anonymous and contains the patient's name.

\section{Conflict of interest}

The authors declare no competing interests.

\section{Publisher's note}

Springer Nature remains neutral with regard to jurisdictional claims in published maps and institutional affiliations.

Supplementary information The online version contains supplementary material available at https://doi.org/10.1038/s41419-021-03685-w.

Received: 26 September 2020 Revised: 20 March 2021 Accepted: 25 March 2021

Published online: 14 April 2021

\footnotetext{
References

1. Kumar, S. K. et al. Multiple myeloma. Nat. Rev. Dis. Prim. 3, 17046 (2017).

2. Siegel, R. L., Miller, K. D. \& Jemal, A. Cancer statistics, 2019. CA 69, 7-34 (2019).
}

3. Neri, P., Bahlis, N. \& Lonial, S. J. C. New strategies in multiple myeloma: immunotherapy as a novel approach to treat patients with multiple myeloma. Clin. Cancer Res. 22, 5959-5965 (2016).

4. Okazuka, K. \& Ishida, T. Proteasome inhibitors for multiple myeloma. Jpn. J. Clin. Oncol. 48, 785-793 (2018).

5. Cengiz Seval, G. \& Beksac, M. A comparative safety review of histone deacetylase inhibitors for the treatment of myeloma. Expert Opin. Drug Saf. 18, 563-571 (2019).

6. Petrucci, M. T. \& Vozella, F. The anti-CD38 antibody therapy in multiple myeloma. Cells 8, https://doi.org/10.3390/cells8121629 (2019).

7. Hosen, N. Chimeric antigen receptor T-cell therapy for multiple myeloma. Cancers 11, https://doi.org/10.3390/cancers11122024 (2019).

8. Soekojo, C. Y. \& Kumar, S. K. Stem-cell transplantation in multiple myeloma: how far have we come? Ther. Adv. Hematol. 10, 2040620719888111 (2019).

9. Chim, C. S. et al. Management of relapsed and refractory multiple myeloma: novel agents, antibodies, immunotherapies and beyond. Leukemia 32, 252-262 (2018)

10. Landgren, O. et al. Monoclonal gammopathy of undetermined significance (MGUS) consistently precedes multiple myeloma: a prospective study. Blood 113, 5412-5417 (2009).

11. Avet-Loiseau, $\mathrm{H}$. et al. Rearrangements of the c-myc oncogene are present in 15\% of primary human multiple myeloma tumors. Blood $\mathbf{9 8}$, 3082-3086 (2001).

12. López-Corral, L. et al. SNP-based mapping arrays reveal high genomic complexity in monoclonal gammopathies, from MGUS to myeloma status. Leukemia 26, 2521-2529 (2012).

13. Walker, B. A. et al. APOBEC family mutational signatures are associated with poor prognosis translocations in multiple myeloma. Nat. Commun. 6, 6997 (2015).

14. Chesi, M. et al. AID-dependent activation of a MYC transgene induces multiple myeloma in a conditional mouse model of post-germinal center malignancies. Cancer Cell 13, 167-180 (2008).

15. Kuzyk, A. \& Mai, S. c-MYC-induced genomic instability. Cold Spring Harb. Perspect. Med. 4, a014373 (2014).

16. Garcia-Gutierrez, L., Delgado, M. D. \& Leon, J. MYC oncogene contributions to release of cell cycle brakes. Genes 10 https:/doi.org/10.3390/genes10030244 (2019).

17. Dejure, F. R. \& Eilers, M. MYC and tumor metabolism: chicken and egg. EMBO J. 36, 3409-3420 (2017).

18. Jovanovic, K. K. et al. Targeting MYC in multiple myeloma. Leukemia 32 , 1295-1306 (2018)

19. Shen, G. et al. Anlotinib: a novel multi-targeting tyrosine kinase inhibitor in clinical development. J. Hematol. Oncol. 11, 120 (2018).

20. Syed, Y. Y. Anlotinib: first global approval. Drugs 78, 1057-1062 (2018).

21. Zhou, A. P. et al. Anlotinib versus sunitinib as first-line treatment for metastatic renal cell carcinoma: a Randomized Phase II Clinical Trial. Oncologist 24, e702-e708 (2019).

22. Sun, Y. et al. Anlotinib for the treatment of patients with locally advanced or metastatic medullary thyroid cancer. Thyroid 28, 1455-1461 (2018).

23. Chi, Y. et al. Safety and efficacy of anlotinib, a multikinase angiogenesis inhibitor, in patients with refractory metastatic soft-tissue sarcoma. Clin. Cancer Res. 24, 5233-5238 (2018)

24. Shan, H. et al. YL064 activates proteasomal-dependent degradation of c-Myc and synergistically enhances the anti-tumor activity of ABT-199 in diffuse large B cell lymphoma. Signal Transduct. Target Ther. 5, 116 (2020).

25. Lomenick, B. et al. Target identification using drug affinity responsive target stability (DARTS). Proc. Natl Acad. Sci. USA 106, 21984-21989 (2009).

26. Chng, W. J. et al. Clinical and biological implications of MYC activation: a common difference between MGUS and newly diagnosed multiple myeloma. Leukemia 25, 1026-1035 (2011).

27. Sears, R. C. The life cycle of C-myc: from synthesis to degradation. Cell cycle $\mathbf{3}$ 1133-1137 (2004).

28. Huang, L. et al. Integrated genomic analysis identifies deregulated JAK/STATMYC-biosynthesis axis in aggressive NK-cell leukemia. Cell Res. 28, 172-186 (2018).

29. Jiao, L. et al. Betulinic acid suppresses breast cancer aerobic glycolysis via caveolin-1/NF-kappaB/C-Myc pathway. Biochem. Pharmacol. 161, 149-162 (2019).

30. Bjorklund, C. C. et al. Rate of CRL4(CRBN) substrate Ikaros and Aiolos degradation underlies differential activity of lenalidomide and pomalidomide in multiple myeloma cells by regulation of c-Myc and IRF4. Blood Cancer J. 5, e354 (2015). 
31. Siu, K. T. et al. Preclinical activity of CPI-0610, a novel small-molecule bromodomain and extra-terminal protein inhibitor in the therapy of multiple myeloma. Leukemia 31, 1760-1769 (2017).

32. Delmore, J. et al. BET bromodomain inhibition as a therapeutic strategy to target c-Myc. Cell 146, 904-917 (2011).

33. Goga, A., Yang, D., Tward, A. D., Morgan, D. O. \& Bishop, J. M. Inhibition of CDK1 as a potential therapy for tumors over-expressing MYC. Nat. Med. 13, 820-827 (2007).

34. Liu, L. et al. Deregulated MYC expression induces dependence upon AMPKrelated kinase 5. Nature 483, 608-612 (2012).

35. Follis, A. V., Hammoudeh, D. I., Wang, H., Prochownik, E. V. \& Metallo, S. J. Structural rationale for the coupled binding and unfolding of the c-Myc oncoprotein by small molecules. Chem. Biol. 15, 1149-1155 (2008).

36. Wang, H., Ramakrishnan, A., Fletcher, S. \& Prochownik, E. V. A quantitative, surface plasmon resonance-based approach to evaluating DNA binding by the c-Myc oncoprotein and its disruption by small molecule inhibitors. J. Biol. Methods 2, https://doi.org/10.14440/jbm.2015.54 (2015).

37. Yao, R. et al. Identification of a novel c-Myc inhibitor with antitumor effects on multiple myeloma cells. Biosci. Rep 38, https://doi.org/10.1042/BSR20181027 (2018).

38. Luo, H. et al. WP1130 reveals USP24 as a novel target in T-cell acute lymphoblastic leukemia. Cancer Cell Int. 19, 56 (2019).

39. Yu, M. et al. Wu-5, a novel USP10 inhibitor, enhances crenolanib-induced FLT3ITD-positive AML cell death via inhibiting FLT3 and AMPK pathways. Acta Pharmacol Sin. 42, 604-612 (2021).

40. Birmann, B. M. et al. Prediagnosis biomarkers of insulin-like growth factor-1, insulin, and interleukin-6 dysregulation and multiple myeloma risk in the Multiple Myeloma Cohort Consortium. Blood 120, 4929-4937 (2012).
41. Lu, A. et al. Inhibition of transforming growth factor-beta activation diminishes tumor progression and osteolytic bone disease in mouse models of multiple myeloma. Am. J. Pathol. 186, 678-690 (2016).

42. Podar, K., Richardson, P. G., Chauhan, D. \& Anderson, K. C. Targeting the vascular endothelial growth factor pathway in the treatment of multiple myeloma. Expert Rev. Anticancer Ther. 7, 551-566 (2007).

43. Kristensen, I. B. et al. Hepatocyte growth factor pathway upregulation in the bone marrow microenvironment in multiple myeloma is associated with lytic bone disease. Br. J. Haematol. 161, 373-382 (2013).

44. Tsubaki, M. et al. Inhibition of the tumour necrosis factor-alpha autocrine loop enhances the sensitivity of multiple myeloma cells to anticancer drugs. Eur. J. Cancer 49, 3708-3717 (2013).

45. Steiner, N. et al. High levels of FLT3-ligand in bone marrow and peripheral blood of patients with advanced multiple myeloma. PLOS ONE 12, e0181487 (2017).

46. Lind, J., Gzernilofsky, F., Vallet, S. \& Podar, K. Emerging protein kinase inhibitors for the treatment of multiple myeloma. Expert Opin. Emerg. Drugs 24, 133-152 (2019).

47. Kimlinger, T. et al. Differential expression of vascular endothelial growth factors and their receptors in multiple myeloma. Haematologica 91, 1033-1040 (2006).

48. Montero, J., López-Pérez, R., San Miguel, J. \& Pandiella, A. J. H. Expression of cKit isoforms in multiple myeloma: differences in signaling and drug sensitivity. haematologica 93, 851-859 (2008).

49. Coluccia, A. et al. Validation of PDGFRbeta and c-Src tyrosine kinases as tumor/ vessel targets in patients with multiple myeloma: preclinical efficacy of the novel, orally available inhibitor dasatinib. Blood 112, 1346-1356 (2008).

50. Ciciarello, M. et al. The Yin and Yang of the bone marrow microenvironment: pros and cons of mesenchymal stromal cells in acute myeloid leukemia. Front Oncol. 9, 1135 (2019).

51. Ria, R. \& Vacca, A. J. I. Bone marrow stromal cells-induced drug resistance in multiple myeloma. Int. J. Mol. Sci. 21, 613 (2020). 